Research Article

\title{
Identification and External Validation of a Transcription Factor-Related Prognostic Signature in Pediatric Neuroblastoma
}

\author{
Rujia Wang (iD) and Qian Wang (iD \\ Department of Pediatrics, Shanghai General Hospital, Shanghai Jiao Tong University School of Medicine, Shanghai, China \\ Correspondence should be addressed to Qian Wang; wangqiansq@njmu.edu.cn
}

Received 10 November 2021; Revised 12 December 2021; Accepted 17 December 2021; Published 28 December 2021

Academic Editor: Song Cao

Copyright ( $) 2021$ Rujia Wang and Qian Wang. This is an open access article distributed under the Creative Commons Attribution License, which permits unrestricted use, distribution, and reproduction in any medium, provided the original work is properly cited.

\begin{abstract}
Background. Neuroblastoma is a common solid tumor originating from the sympathetic nervous system, commonly found in children, and it is one of the leading causes of tumor-related deaths in children. In addition to pathological features, molecularlevel features, such as how much gene expression is present and the mutational profile, may provide useful information for the precise treatment of neuroblastoma. Transcription factors (TFs) play an important regulatory role in all aspects of cellular life activities. But there are currently no studies on transcription factor-based biomarkers of neuroblastoma prognosis, and this study is much needed. Methods. We downloaded RNA transcriptome data and clinical data from the TARGET database to construct a prognostic model. The prognostic model was constructed by using univariate Cox analysis, LASSO, and multivariate Cox regression. We divided the patients into low-risk and high-risk groups using the median value of the risk score as the cut-off. Then, we validated the prognostic model with the dataset GSE49710. Results. We constructed a prognostic model consisting of eight genes (SATB1, ZNF564, SOX14, EN1, IKZF2, SLC2A4RG, FOXJ2, and ZNF521). Patients in the high-risk group had a lower survival rate than those in the low-risk group. The area under the 3-year ROC curve of the model reached 0.825 , suggesting a good predictive efficacy. We performed target gene prediction for the eight transcription factors in the model using six online databases and found that TUT1 may be a target gene for transcription factor EN1 and is associated with immune infiltration. Conclusion. This prognostic model consisting of eight transcription factor-associated genes demonstrated reliable predictive efficacy. This prediction model may provide new potential targets for the treatment of neuroblastoma and personalized monitoring of neuroblastoma patients with high and low risk.
\end{abstract}

\section{Introduction}

Neuroblastoma is the most common extracranial solid tumor of childhood, accounting for $8 \%$ of all pediatric tumors and also $15 \%$ of tumor-related deaths in children [1]. Neuroblastoma arises from neural crest precursor cells, which proliferate uncontrollably due to impaired differentiation [2]. The clinical diversity of neuroblastoma reflects its heterogeneous character. In some patients, the tumor regresses on its own, while others progress rapidly and become resistant to treatment. Although significant progress has been made in the treatment of neuroblastoma in recent years, recent studies have identified several molecules, such as MYCN, ALK, and ARID1B, involved in the development of neuroblastoma [3], the prognosis of neuroblastoma patients remains very poor, especially in cases of advanced or recurrent tumors. Therefore, there is an urgent need to discover novel markers with prognostic and predictive power in order to target and individualize the treatment of neuroblastoma patients.

Transcription factors (TFs) are a number of proteins that can specifically bind DNA sequences and regulate transcription [4]. TFs play an important role in tumorigenesis, progression, invasion, metastasis, and drug resistance [5]. Statistically, about $20 \%$ of oncogenes are TFs [6]. On the other hand, loss of function of TFs with tumorigenic 
suppressive effects leads to uncontrolled cell division and cancer development and progression [7]. However, there are few studies on TFs with prognostic values.

In this study, we constructed a prognostic model for patients with neuroblastoma based on TF-related genes by downloading transcriptomic data from the Therapeutically Applicable Research to Generate Effective Treatments (TARGET) database and validated it using the Gene Expression Omnibus (GEO) database. Our study found that our risk score could be an independent prognostic factor for neuroblastoma. In conclusion, our study shows that the prognostic model has a very high predictive value for neuroblastoma patients and provides a new potential target for the treatment of neuroblastoma patients.

\section{Materials and Methods}

2.1. Data Collection. In 2018, Lambert et al. published a review that identified more than 1600 TFs that may be involved in human physiological and disease processes [8]. We collected 1639 TFs from the literature for analysis (Table S1). Figure 1 shows the flow chart of this study. We downloaded transcriptomic data and clinical information from the TARGET database and the GEO database. Data from 148 patients in the TARGET database were used for model construction. Data from 498 neuroblastoma patients in the GEO dataset (GSE49710) were used for model validation.

2.2. Construction of the Prognostic TF-Based Signature. To screen TFs with prognostic value, we performed univariate Cox regression analysis of 1639 TFs to assess the correlation between TFs and overall survival (OS) in the TARGET dataset. We defined a screening criterion of Cox $P<0.01$ for later analysis. To avoid overfitting between variables during model construction, we used the least absolute shrinkage and selection operator (LASSO) regression algorithm to extract the best subset. Finally, we obtained the coefficients of each incorporated TF in the model. Risk scores were calculated according to the following equation:

$$
\text { risk score }=\sum_{i=1}^{N}(\text { Expi } * \text { Coei }) \text {. }
$$

Expi represents the expression of each TF, and Coei is the regression coefficient of the corresponding multivariate Cox result.

To further validate the predictive performance of the TFbased prognostic model, we evaluated the AUC of the timedependent ROC curves to assess the predictive value of risk scores on time-dependent outcomes.

2.3. Gene Set Enrichment Analysis (GSEA). We used GSEA to analyze the potentially different biological mechanisms between the high- and low-risk groups. The GSEA was annotated using the "clusterProfiler" package in $\mathrm{R}$ software.
2.4. Immune Infiltration Analysis. We calculated the level of 22 tumor-infiltrating immune cells by CIBERSORT algorithm [9], a method to calculate a certain cell composition from the gene expression profile of a tissue. In the present study, we used the CIBERSORT algorithm to calculate the level of immune cell infiltration in tumor samples from the TARGET and GEO databases.

2.5. Statistical Analysis. We used R software (version 3.8.2) to complete all statistical analyses. Student's $t$-test was employed to examine statistically significant differences between groups, while one-way ANOVA was used for the comparison of differences between groups. The chi-square test was employed to compare the clinical features of the two groups. Neuroblastoma patients were analyzed using the Kaplan-Meier technique and the log-rank test to determine their overall survival time (OS). The predictive value of risk score and clinical characteristics was assessed by using oneway and Cox regression analysis. Spearman analysis was used to analyze the correlation between the two variables $(P<0.05)$.

\section{Results}

3.1. Identifying the Potential Prognostic Transcription Factor. We obtained a list of 1639 human TFs from the public literature $[8,10]$. After corresponding to the TARGET database, a total of 1375 TFs had gene expression data for inclusion in the follow-up study. Information on the clinical characteristics of neuroblastoma patients in both datasets is in Table S2.

Univariate Cox regression analysis of the gene expression was performed, and we searched for TFs with prognostic significance from 1375 TFs. 65 TFs with $P \leq 0.01$ were obtained for the next analysis (Table S3).

3.2. Constructing the TF-Based Predictive Model. To avoid overfitting between variables during model construction, we used the LASSO regression algorithm to extract the best subset (Figure 2). Then, we obtained the coefficients of each $\mathrm{TF}$ in the prognostic model by multivariate Cox regression analysis (Table 1), consisting of 8 genes that constituted this prognostic model. The coefficients of ZNF564, SOX14, EN1, and SLC2A4RG were positive and were risk genes for poor prognosis. In contrast, the coefficients of SATB1, IKZF2, FOXJ2, and ZNF521 were negative and were protective factors.

We divided the patients into high- and low-risk groups by median risk score (Figure 3(c)). High-risk patients had a higher mortality rate than low-risk patients (Figure 3(b)). From the heat map, we found that ZNF564, SOX14, EN1, and SLC2A4RG had increased expression in high-risk group, while SATB1, IKZF2, FOXJ2, and ZNF521 had increased expression in the low-risk group (Figure 3(e)). Figure 3(a) shows the Kaplan-Meier curves for the two groups, with longer OS in low-risk patients than the other groups. Our sensitivity and specificity evaluation of the model was performed by time-dependent ROC analysis. In 


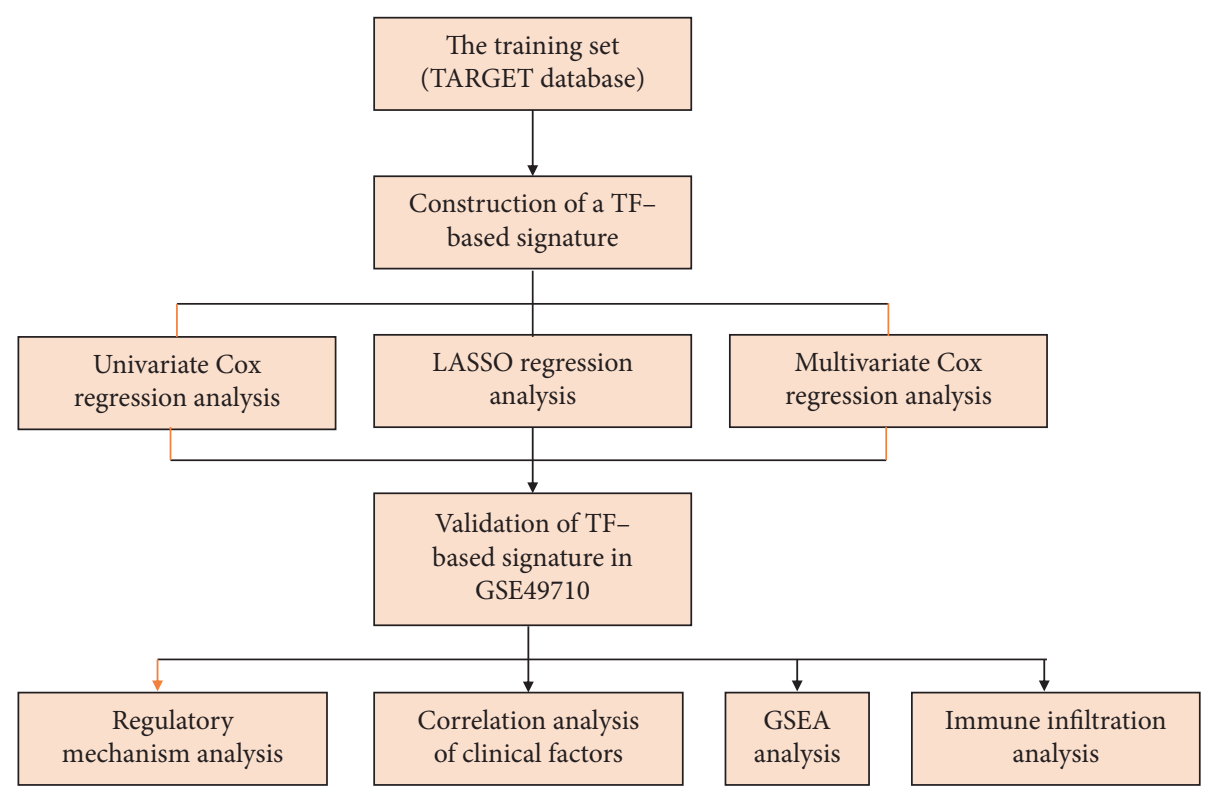

FIgURe 1: Flowchart of this study.

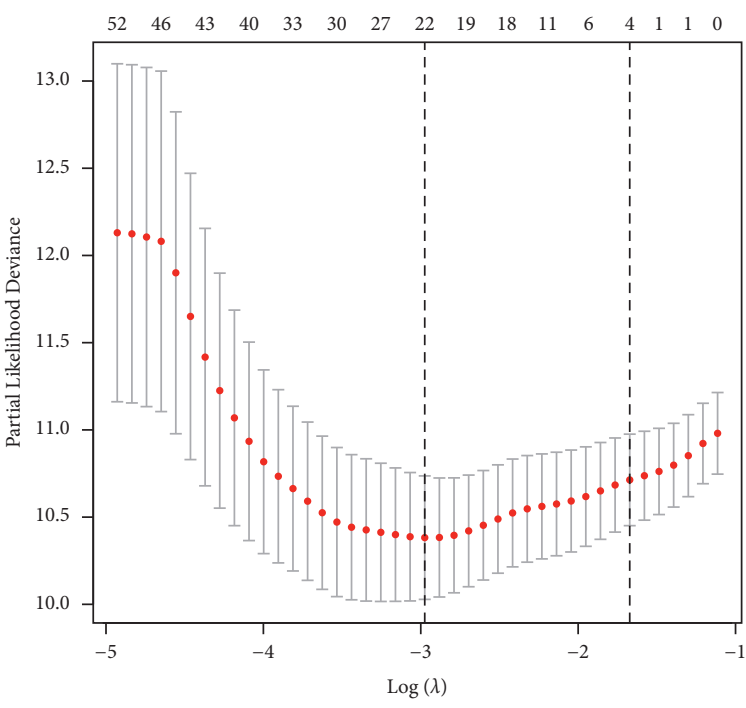

(a)

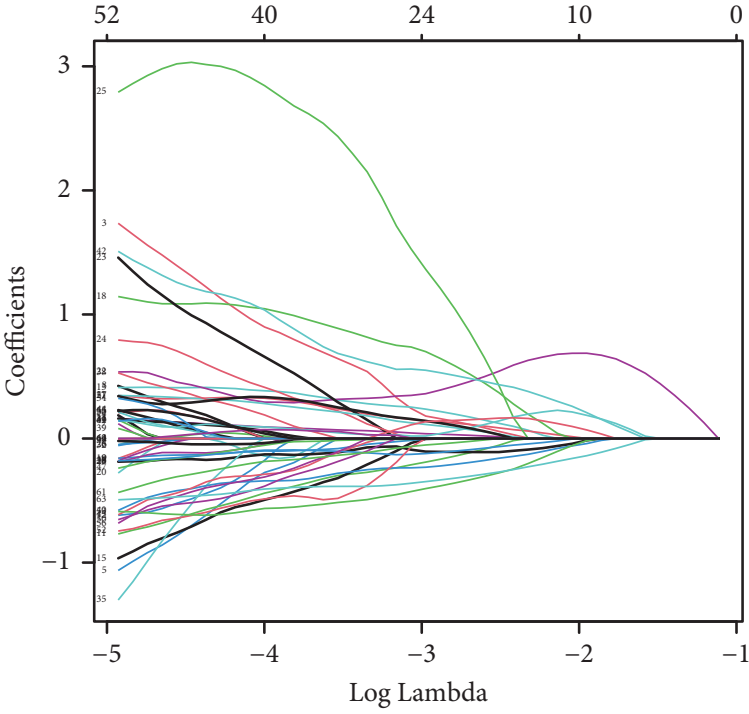

(b)

FIGURE 2: 65 TFs with significant prognostic value subjected to LASSO regression analysis. (a) Each line represents a TF with a significant prognostic value. (b) Plot of partial likelihood deviation.

the 3-year ROC curve, the AUC was 0.825 , indicating the good predictive performance of the prediction model for 3year OS (Figure 3(d)).

3.3. Validation of the TF-Based Signature. The prediction model we constructed was validated in another dataset GSE49710. We used the same prognostic model obtained from the TARGET dataset to calculate risk scores for a total of 498 patients in the GSE49710 dataset. The same division into low- and high-risk groups was done according to the median risk score. In GSE49710, the sensitivity and specificity of our TF-based prognostic model for 3-year OS were evaluated as 0.778 (Figure 4(c)). Other findings, including heat map and Kaplan-Meier analysis, were also consistent with the results of the TARGET cohort, suggesting that our TF-based prognostic model has good stability in neuroblastoma patients (Figures 4(a), 4(b), 4(d), and $4(\mathrm{e}))$.

\subsection{The Identification of the TF-Based Signature's Independent} Predictive Activity. To further investigate whether the TFbased prognostic model can independently predict the prognosis of neuroblastoma patients. Univariate Cox regression analysis of whether the TF-based prognostic model 
TABLE 1: Eight TF-related genes identified by multivariate Cox regression analysis.

\begin{tabular}{lcccrr}
\hline Id & coef & HR & HR.95L & HR.95H & $P$ value \\
\hline SATB1 & -0.56897 & 0.566111 & 0.365756 & 0.876216 & 7.258708 \\
ZNF564 & 1.370636 & 3.937853 & 2.136288 & 271.6841 & $1.12 \mathrm{E}-05$ \\
SOX14 & 3.153827 & 23.42554 & 2.019831 & 1.684837 & 0.011663 \\
EN1 & 0.34383 & 1.410339 & 1.180563 & 0.720567 & 0.000151 \\
IKZF2 & -0.87769 & 0.415742 & 0.239868 & 0.001761 \\
SLC2A4RG & 1.118871 & 3.061396 & 1.680095 & 0.578342 & 0.000257 \\
FOXJ2 & -0.65706 & 0.518375 & 0.313321 & 0.857627 & 0.010532 \\
ZNF521 & -0.66938 & 0.512027 & 0.383883 & 0.682947 & $5.24 \mathrm{E}-06$ \\
\hline
\end{tabular}

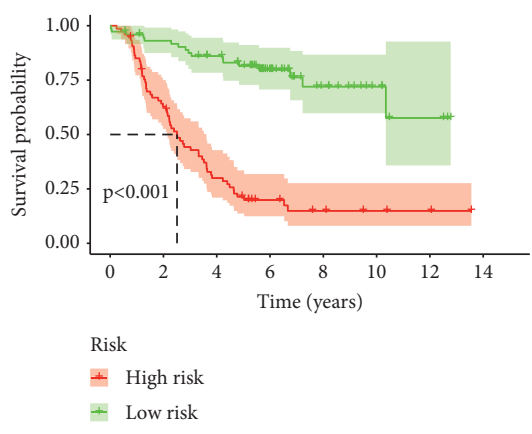

(a)

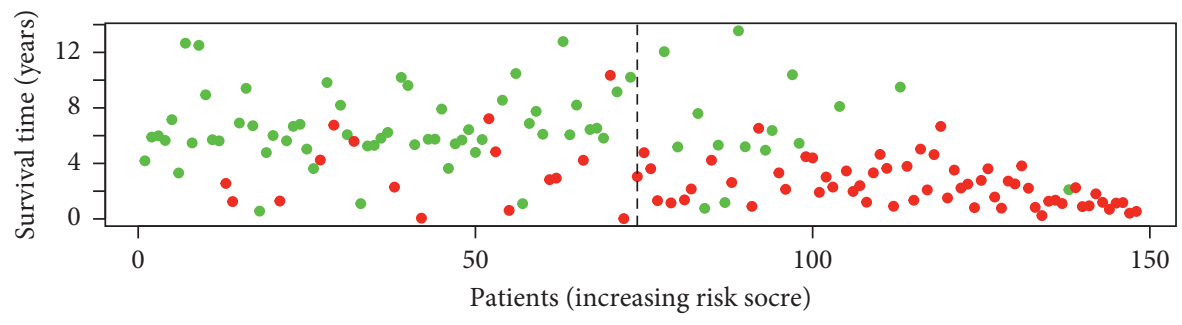

- Dead

- Alive

(b)

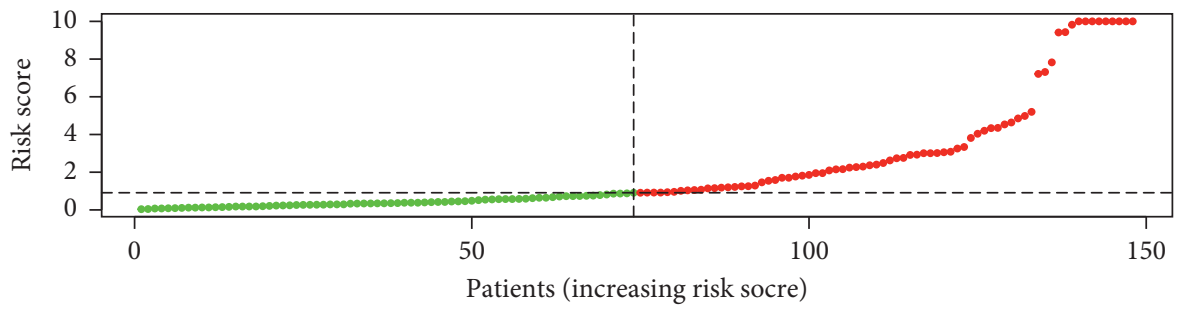

- High risk

- Low Risk

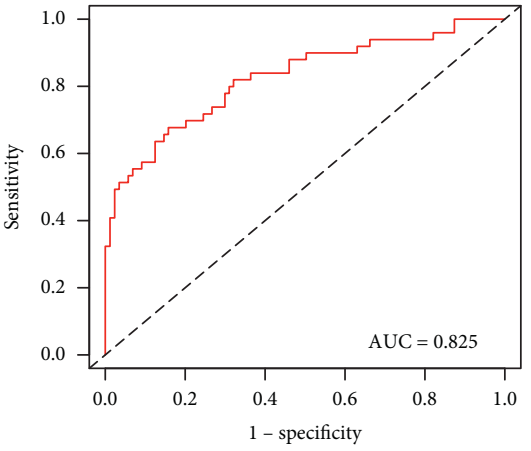

(d)

Figure 3: Continued. 


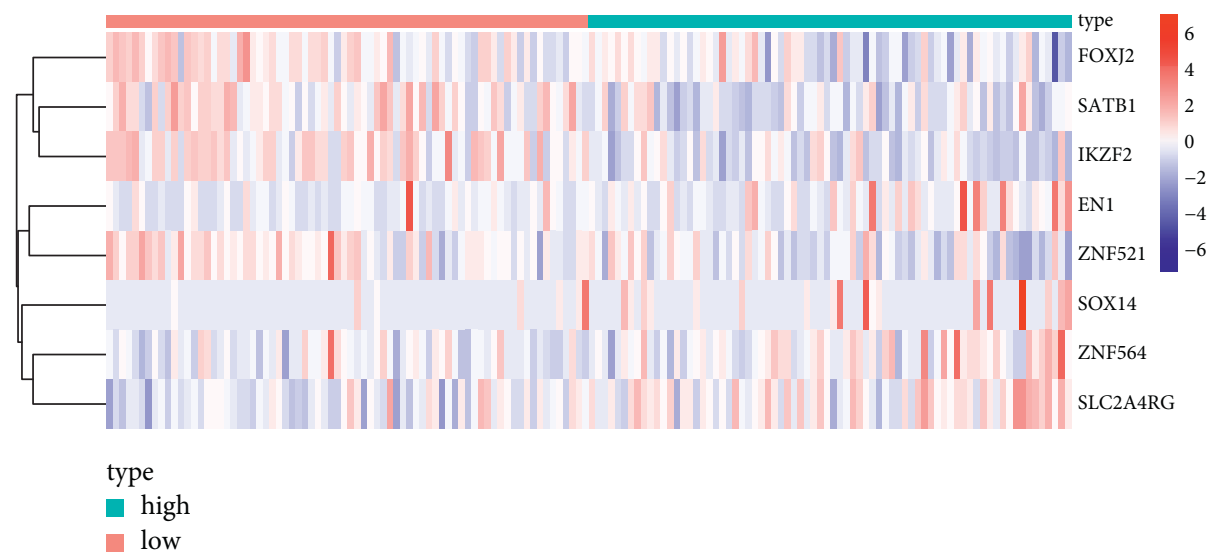

(e)

FIGURE 3: Characteristics of the prognostic model consisting of eight TFs in the TARGET dataset. (a) Kaplan-Meier survival analysis of the TF-based prognostic models. (b) Distribution of survival times of neuroblastoma patients. (c) Distribution of risk scores of neuroblastoma patients. (d) The AUC value reflects the high prognostic accuracy of the TF-based signature. (e) Distribution of the expression values of TFs in the model.

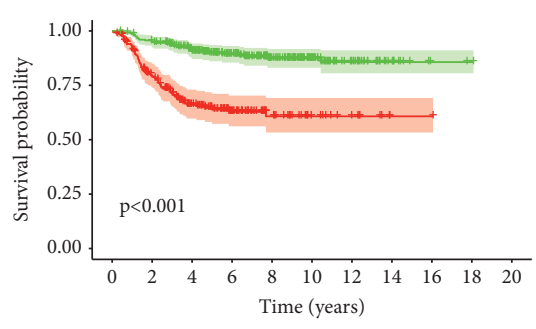

Risk

+ High risk

- Low risk

(a)

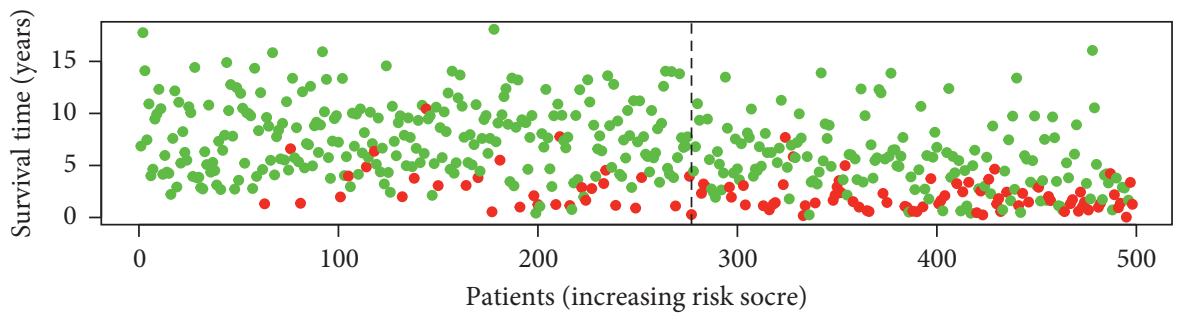

- Dead

- Alive

(b)

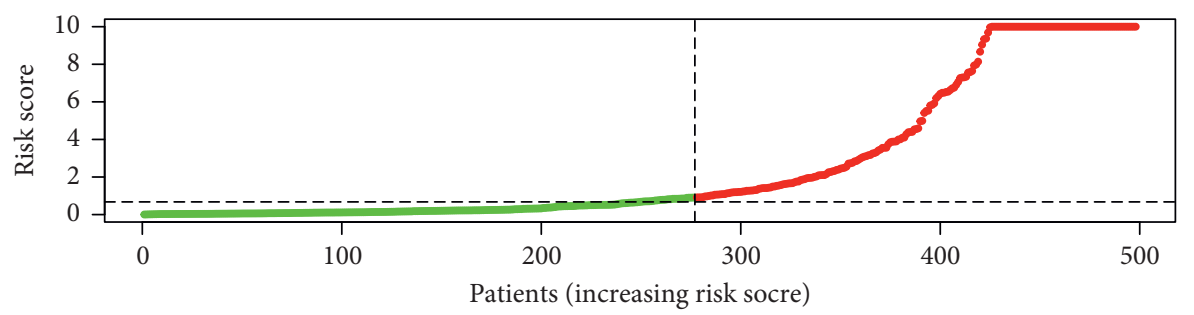

- High risk

- low Risk

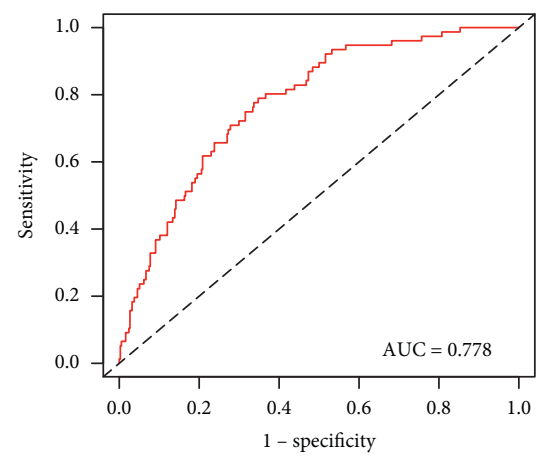

(c)

(d)

Figure 4: Continued. 


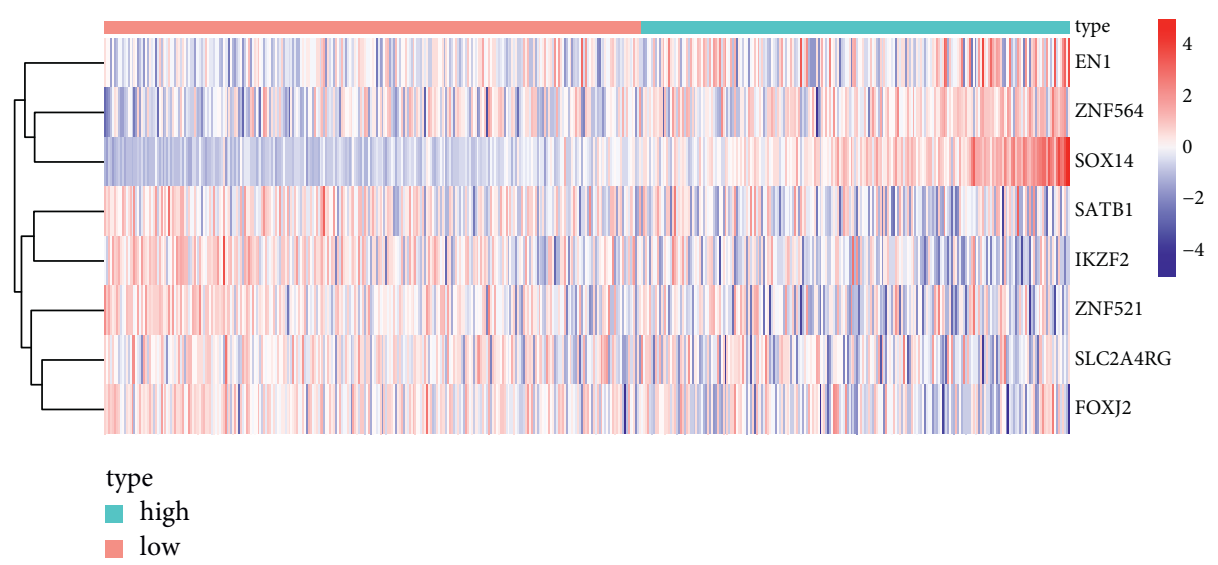

(e)

FIgURE 4: Validation of the eight TF-based risk signatures in GEO cohort. (a) Kaplan-Meier survival analysis of the TF-based prognostic models. (b) Distribution of survival times of neuroblastoma patients. (c) Distribution of risk scores of neuroblastoma patients. (d) Area under the curve reflects the high prognostic accuracy of the TF-based signature. (e) Distribution of the expression values of TFs in the model.

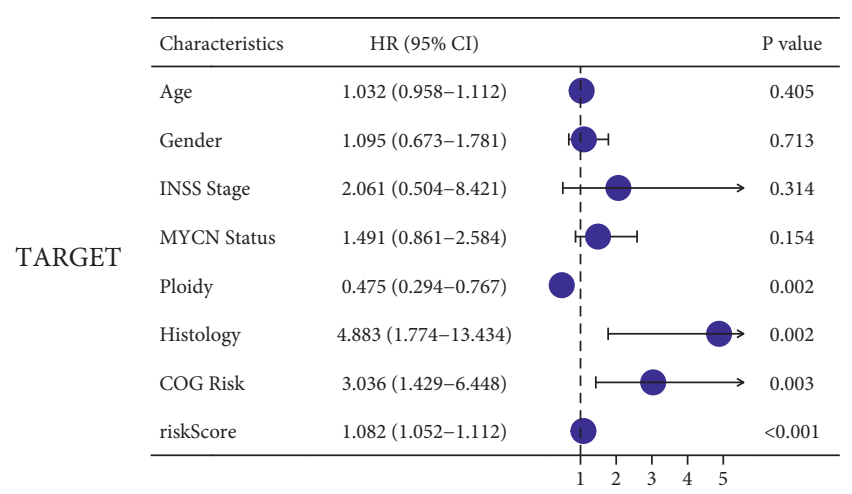

(a)

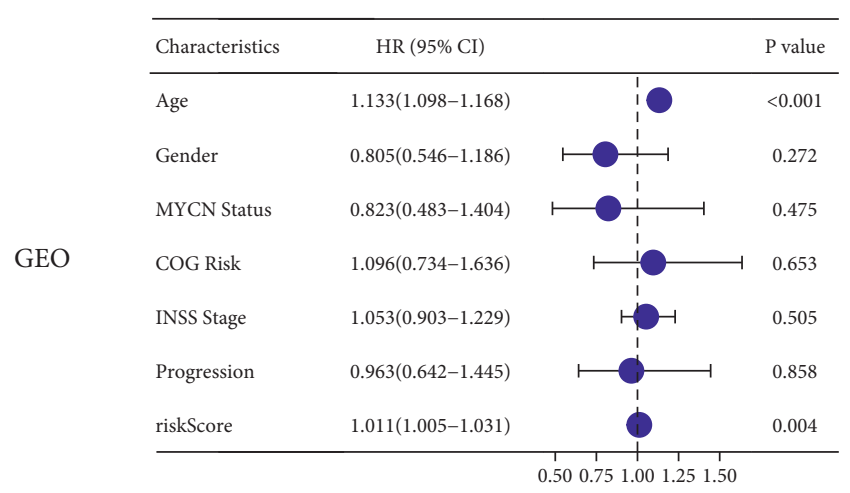

(c)

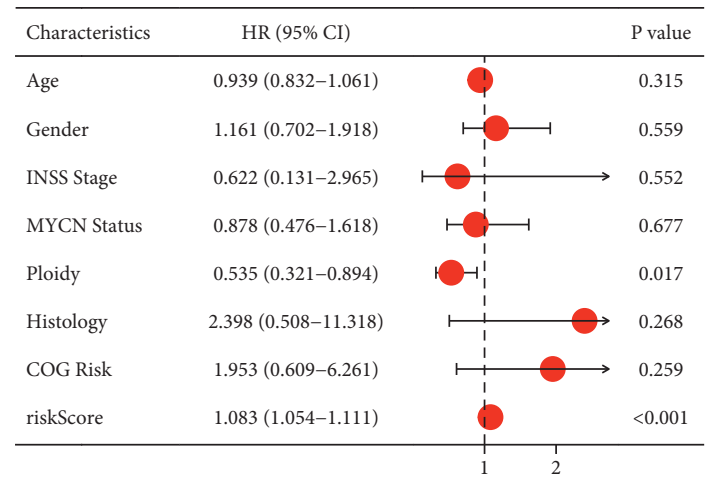

(b)

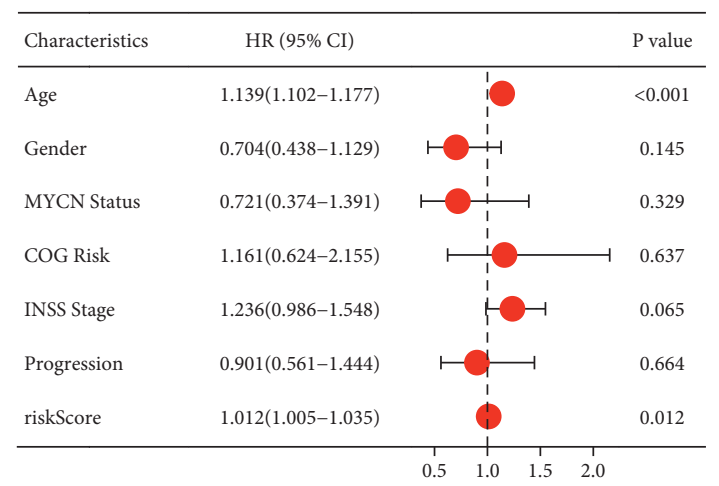

(d)

Figure 5: Univariate $(a, c)$ and multivariate $(b, d)$ Cox regression analyses in OS of neuroblastoma patients among the gene signature and clinicopathological factors. (a, b) TARGET cohort. (c, d) GEO cohort.

could independently predict other clinicopathological characteristics showed that ploidy, histology, COG risk, and risk score were associated with OS in the TARGET dataset (Figure 5(a)). We then included the variables in a multivariate Cox regression analysis showing that ploidy and risk score remained independent predictors of OS (Figure 5(b)). In GSE49710, age and risk score were associated with OS (Figure 5(c)), and age and risk score remained independent prognostic indicators of OS after multivariate Cox regression (Figure 5(d)).

3.5. GSEA Identifies Biological Pathways. To further investigate the relevant signaling pathways in the high-risk group, we performed GSEA analysis between the high-risk and lowrisk groups. In the high-risk group of the TARGET database, 


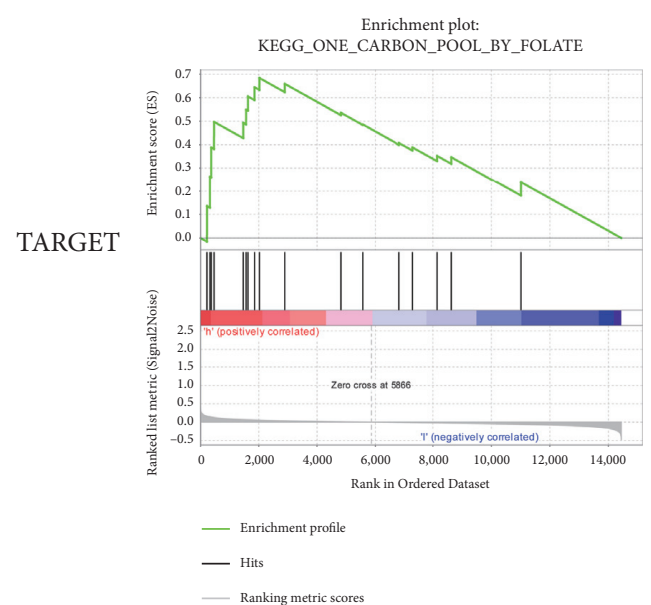

(a)

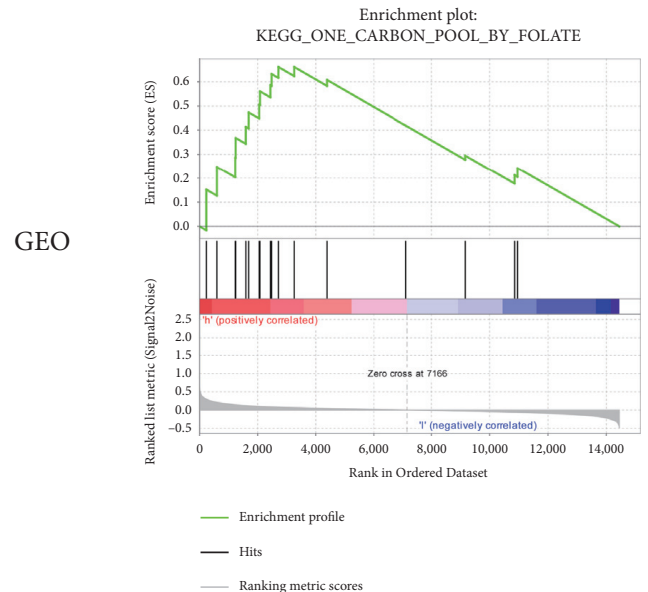

(d)
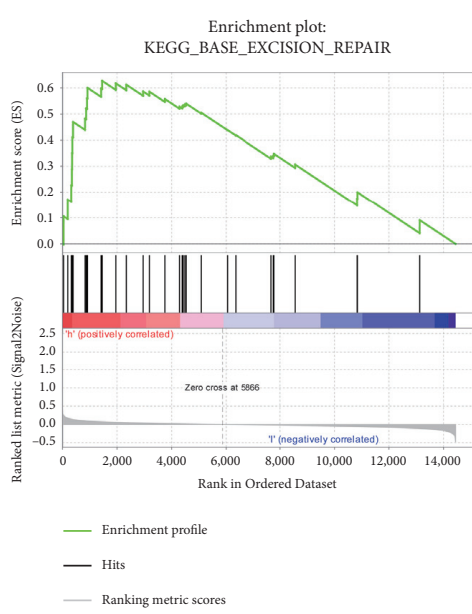

(b)

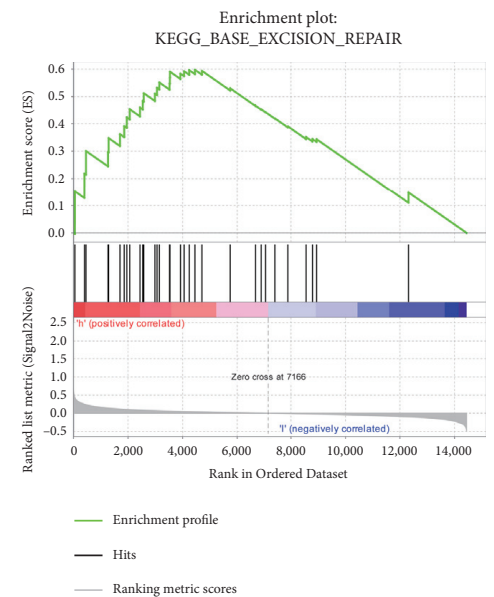

(e)

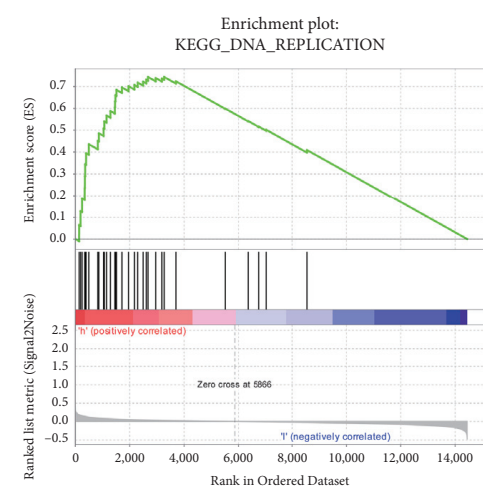

— Enrichment profile

- Hits

- Ranking metric scores

(c)

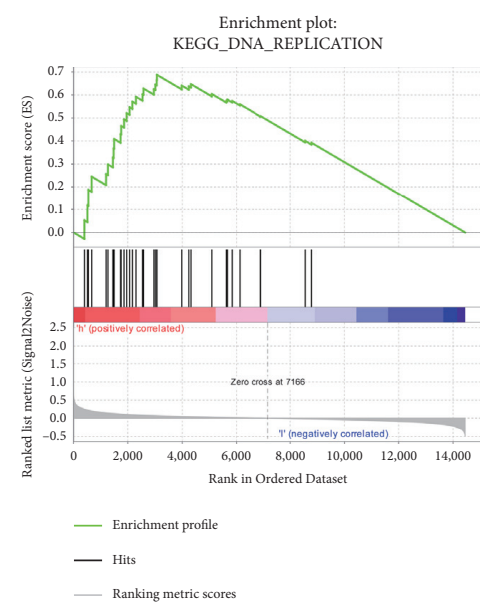

(f)

FIGURE 6: GSEA analysis of the high-risk group in both TARGET cohort (a-c) and GEO cohort (d-f).

TABLE 2: Predictions for the target genes of the eight transcription factors included in the model supported by more than two public databases.

\begin{tabular}{lccccccc}
\hline TF & Gene & CHEA & ENCODE & JASPAR & MotifMap & TRANSFAC & TRRUST \\
\hline EN1 & TUT1 & - & - & $\sqrt{ }$ & - & - & - \\
EN1 & SAV1 & - & - & $\sqrt{ }$ & - & $\sqrt{ }$ & - \\
EN1 & PAX6 & - & - & $\sqrt{ }$ & - & $\sqrt{ }$ & - \\
EN1 & ATP13A4 & - & - & $\sqrt{ }$ & - & $\sqrt{ }$ & - \\
\hline
\end{tabular}

one carbon pool by folate, base excision repair, and DNA replication were enriched (Figures 6(a)-6(c)). These were further validated in the GEO database (Figures 6(d)-6(f)).

3.6. The Regulatory Mechanism Analysis Based on Six Databases. To validate this transcriptional regulatory relationship, we used six online databases, including JASPAR, ENCODE, CHEA, MotifMap, TRANSFAC, and TRRUST. We made predictions for the target genes of the eight TFs in the model (Table S4), listing evidence supported by more than two databases (Table 2). " $\sqrt{ }$ " indicated that the corresponding TF has a regulatory relationship with the target gene.
3.7. Survival Analysis of TUT1 and Correlation with Immune Infiltration. Interestingly, we found that the higher the expression of TUT1, the shorter the OS of the patients in both TARGET and GEO datasets (Figures 7(a) and 7(b)). By using CIBERSORT to calculate immune cell infiltrations, we found that TUT1 was positively correlated with T cells CD8, T cells regulatory (Tregs), macrophages $\mathrm{M} 0, \mathrm{~B}$ cells naive and negatively correlated with $\mathrm{T}$ cells $\mathrm{CD} 4$ memory resting, macrophages M2, monocytes, eosinophils, NK cells resting, dendritic cells activated, and T cells gamma delta in TARGET database (Figure 7(c)). We also found that TUT1 was positively correlated with plasma cells and neutrophils and negatively correlated with eosinophils, dendritic cells resting, T cells CD4 memory resting, and monocytes in GEO database (Figure 7(d)). There may be a role for TUT1 in the 


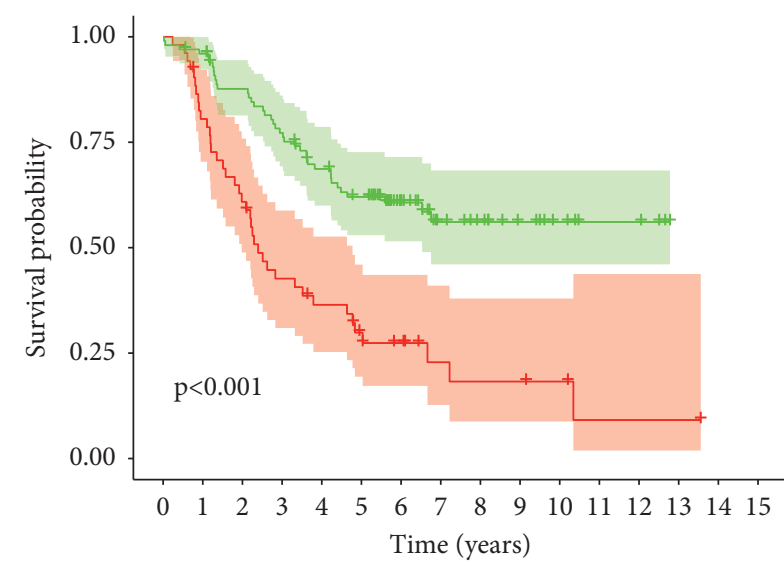

TUT1
+ High
+ Low

(a)

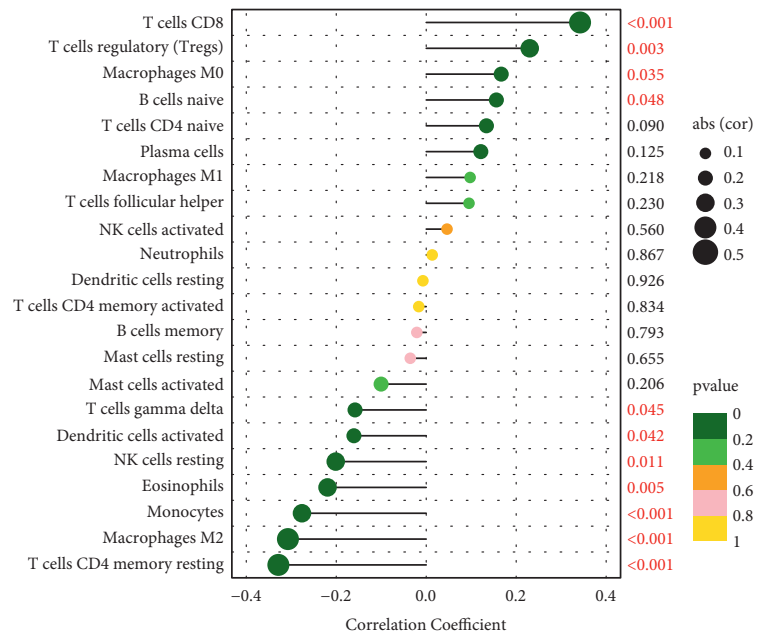

(c)

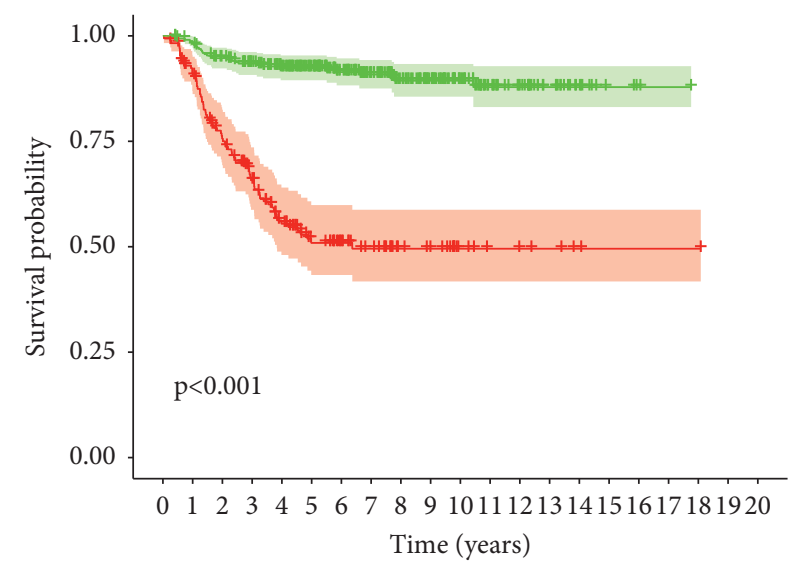

TUT1

* High

+ Low

(b)

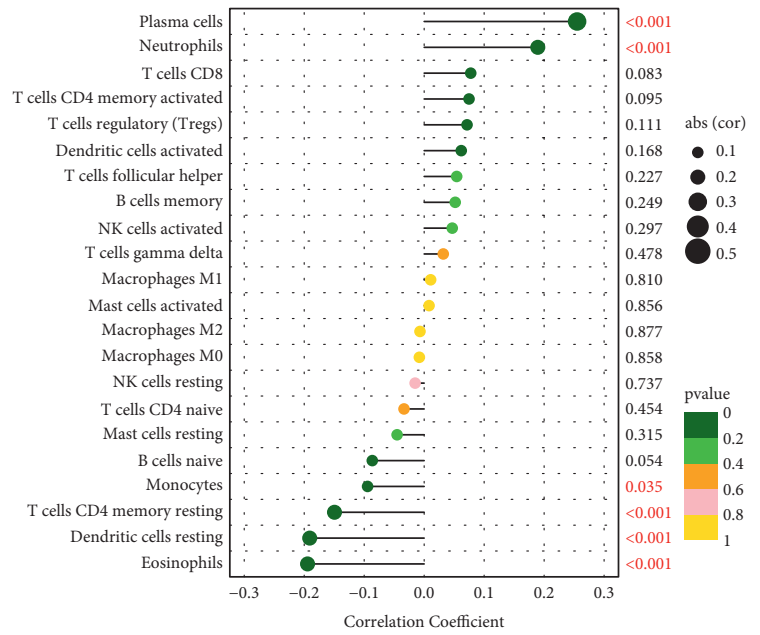

(d)

FIGURE 7: Survival analysis of TUT1 and correlation between TUT1 and infiltration immune cells. (a, c) TARGET cohort. (b, d) GEO cohort.

development of neuroblastoma, and the transcription factor EN1 may also be regulated in relation to the predicted target gene TUT1. This needs to be further elucidated.

\section{Discussion}

In this study, we showed that the TFs-based prognostic model we constructed could predict neuroblastoma patients well. TFs have a role in gene transcription as well as a variety of other critical biological activities [11, 12]. A third of all human developmental diseases are linked to aberrant TF expression [13]. Dong et al. discovered that transcription factors might enhance breast cancer development [14]. A crucial involvement for transcription factors in hepatoblastomas was found by Zhan and colleagues [15]. TFs have also been implicated with neuroblastoma in several investigations [16]. However, there are few studies on the prognostic aspects of transcription factors. As effector molecules of cell signaling pathways, TFs play an important role in tumor development, and therefore, it is necessary to study their functions in predicting patient prognosis. We analyzed data from TARGET and GEO databases by bioinformatics methods to construct and validate the role of prognostic models and analyze their association with clinical features and immune infiltration and also predict target genes of transcription factors. We used the data from TARGET for model construction and the dataset from GEO for model validation based on 8 TFs using Cox regression and LASSO regression methods. This signature can well predict the prognosis of patients with neuroblastoma.

In this study, a prognostic model consisting of eight TFs whose roles in tumors have been partially reported was constructed. SATB1 is capable of regulating chromatin structure and gene expression through chromatin remodeling enzymes. SATB1 is expressed in a diverse population of adult progenitor cells and embryonic stem cells. This gene has been linked to a number of various forms of cancers, such as laryngeal squamous-cell carcinoma [17], endometrial cancer [18], hepatocellular 
carcinoma [19, 20], rectal cancer [21], melanoma [22], and gastric cancer $[23,24]$. SOX14 is a member of the SOXB2 transcription factor subgroup, and $\mathrm{Li}$ et al. reported that through the Wnt/-catenin pathway [25], SOXQ4 might enhance cervical cancer cell proliferation and invasion. EN1 plays a role in abnormal expression of EN1 is common in colorectal cancer [26], prostate cancer [27], and astrocytoma [28]. IKZF2 is a member of the Ikaros family of transcription factors and has been shown to be a transcription factor essential for regulatory $\mathrm{T}$-cell function $[29,30]$. Park et al. reported that IKZF2 could inhibit myeloid differentiation by driving the self-renewal of leukemic stem cells [31]. It has been shown by Zhao et al. that SLC2A4RG encodes a nuclear transcription factor that helps activate the solute carrier family two member four gene, a gene that may have a role in the formation of GBM [32]. In addition to being a member of the FOX family, FOXJ2 is a new forkhead factor with dual DNA binding specificity. Breast cancer cell migration and invasion may be inhibited if FOXJ2 expression is increased [33]. ZNF521 is a transcription factor involved in the regulation of hematopoietic, neural, and mesenchymal stem cells, and Chiarella et al. reported that ZNF521 inhibits the differentiation of human adipose-derived stem cells [34]. These studies suggest that these transcription factors are closely associated with tumors. Our prognostic model can well predict the prognosis of neuroblastoma patients.

We also used six databases to predict our eight TF target genes and found that the TUT1 gene not only has prognostic value but was found to be associated with immune cells by the CIBERSORT algorithm, which was validated in both the TARGET database and the GEO database. This gene encodes a nucleotidyltransferase that functions as both a terminal uridylyltransferase and a nuclear poly(A) polymerase. The encoded enzyme specifically adds and removes nucleotides from the $3^{\prime}$ end of small nuclear RNAs and select mRNAs and may function in controlling gene expression and cell proliferation. This provides a direction for further research in the future. Although our model was able to predict the prognosis of neuroblastoma patients well, our study still has some shortcomings. Our prognostic model still needs clinical cases for further validation, not just using data on public databases. Some of the TFs in our model has not been studied in neuroblastoma with relevant mechanisms, which need to be refined in our future studies.

\section{Conclusion}

Our study successfully constructed a prognostic model containing eight TFs (ZNF564, SOX14, EN1, SLC2A4RG, SATB1, IKZF2, FOXJ2, and ZNF521). Our prognostic model can help clinicians predict OS in neuroblastoma patients, but further studies with clinical samples are needed to validate the accuracy of our prognostic model to improve the management of neuroblastoma.

\section{Data Availability}

All of the data used in this study are available online.

\section{Conflicts of Interest}

The authors declare that there are no conflicts of interest.

\section{Authors' Contributions}

RW designed the project and participated in the data analysis. QW interpreted the data, wrote the manuscript, and supervised the project. All authors contributed to the article and approved the submitted version.

\section{Acknowledgments}

This work was supported by the Medical-Engineering Intersection Program of Shanghai Jiao Tong University (Grant no. YG2021QN83).

\section{Supplementary Materials}

Table S1: 1639 TFs from public literature. Table S2: clinical and pathologic factors of the datasets used in this study. Table S3: 65 TFs with $P \leq 0.01$ after univariate Cox regression. Table S4: predictions for the target genes of the eight TFs. (Supplementary Materials)

\section{References}

[1] E. A. Newman, S. Abdessalam, J. H. Aldrink et al., "Update on neuroblastoma," Journal of Pediatric Surgery, vol. 54, no. 3, pp. 383-389, 2019.

[2] I. S. Fetahu and S. Taschner-Mandl, "Neuroblastoma and the epigenome," Cancer and Metastasis Reviews, vol. 40, no. 1, pp. 173-189, 2021.

[3] M. Huang and W. A. Weiss, "Neuroblastoma and MYCN," Cold Spring Harbor Perspectives in Medicine, vol. 3, no. 10, 2013.

[4] D. L. Fulton, S. Sundararajan, G. Badis et al., "TFCat: the curated catalog of mouse and human transcription factors," Genome Biology, vol. 10, no. 3, p. R29, 2009.

[5] K. Vishnoi, N. Viswakarma, A. Rana, and B. Rana, "Transcription factors in cancer development and therapy," Cancers, vol. 12, no. 8, p. 2296, 2020.

[6] J. H. Bushweller, "Targeting transcription factors in cancer-from undruggable to reality," Nature Reviews Cancer, vol. 19, no. 11, pp. 611-624, 2019.

[7] W. Sun and J. Yang, "Functional mechanisms for human tumor suppressors," Journal of Cancer, vol. 1, pp. 136-140, 2010.

[8] S. A. Lambert, A. Jolma, L. F. Campitelli et al., "The human transcription factors," Cell, vol. 172, no. 4, pp. 650-665, 2018.

[9] A. M. Newman, C. L. Liu, M. R. Green et al., "Robust enumeration of cell subsets from tissue expression profiles," Nature Methods, vol. 12, no. 5, pp. 453-457, 2015.

[10] J. M. Vaquerizas, S. K. Kummerfeld, S. A. Teichmann, and N. M. Luscombe, "A census of human transcription factors: function, expression and evolution," Nature Reviews Genetics, vol. 10, no. 4, pp. 252-263, 2009.

[11] B. D. Dynlacht, "Regulation of transcription by proteins that control the cell cycle," Nature, vol. 389, no. 6647, pp. 149-152, 1997.

[12] D. Accili and K. C. Arden, "FoxOs at the crossroads of cellular metabolism, differentiation, and transformation," Cell, vol. 117, no. 4, pp. 421-426, 2004. 
[13] S. Boyadjiev and E. Jabs, "Online mendelian inheritance in man (OMIM) as a knowledgebase for human developmental disorders," Clinical Genetics, vol. 57, no. 4, pp. 253-266, 2000.

[14] G. Dong, G. Ma, R. Wu et al., "ZFHX3 Promotes the proliferation and tumor growth of ER-positive breast cancer cells likely by enhancing stem-like features and MYC and TBX3 transcription," Cancers, vol. 12, no. 11, p. 3415, 2020.

[15] X. Zhan and A. Zhao, "Transcription factor FOXA3 promotes the development of hepatoblastoma via regulating HNF1A, AFP, and ZFHX3 expression," Journal of Clinical Laboratory Analysis, vol. 35, no. 3, Article ID e23686, 2021.

[16] J. De Wyn, M. W. Zimmerman, N. Weichert-Leahey et al., "MEIS2 is an adrenergic core regulatory transcription factor involved in early initiation of TH-MYCN-driven neuroblastoma formation," Cancers, vol. 13, no. 19, p. 4783, 2021.

[17] X. D. Zhao, W. Y. Ji, W. Zhang et al., "Overexpression of SATB1 in laryngeal squamous cell carcinoma," Orl, vol. 72, no. 1, pp. 1-5, 2010.

[18] N. M. Mokhtar, N. H. Ramzi, W. Yin-Ling, I. M. Rose, A. Z. Hatta Mohd Dali, and R. Jamal, "Laser capture microdissection with genome-wide expression profiling displayed gene expression signatures in endometrioid endometrial cancer," Cancer Investigation, vol. 30, no. 2, pp. 156-164, 2012.

[19] W. Tu, M. Luo, Z. Wang et al., "Upregulation of SATB1 promotes tumor growth and metastasis in liver cancer," Liver International, vol. 32, no. 7, pp. 1064-1078, 2012.

[20] Y. K. Huang, X. G. Fan, F. Qiu, and Z. M. Wang, "Genomics of hepatitis B virus-related hepatocellular carcinoma and adjacent noncancerous tissues with cDNA microarray," Chinese Medical Journal, vol. 124, pp. 2057-2064, 2011.

[21] W. J. Meng, H. Yan, B. Zhou et al., "Correlation of SATB1 overexpression with the progression of human rectal cancer," International Journal of Colorectal Disease, vol. 27, no. 2, pp. 143-150, 2012.

[22] H. Chen, M. Takahara, J. Oba et al., "Clinicopathologic and prognostic significance of SATB1 in cutaneous malignant melanoma," Journal of Dermatological Science, vol. 64, no. 1, pp. 39-44, 2011.

[23] I. Calico, C. Juste, L. Arcalis, S. M. Morales, and S. F. Castillo, "[Toxoplasmosis diagnosed using virologic methods]," Enfermedades Infecciosas Microbiologia Clinica, vol. 8, pp. 392-393, 1990.

[24] C. Cheng, X. Lu, G. Wang et al., "Expression of SATB1 and heparanase in gastric cancer and its relationship to clinicopathologic features," Apmis, vol. 118, no. 11, pp. 855-863, 2010.

[25] F. Li, T. Wang, and S. Tang, "SOX14 promotes proliferation and invasion of cervical cancer cells through Wnt/beta-catenin pathway," International Journal of Clinical Experimental Pathology, vol. 8, pp. 1698-1704, 2015.

[26] R. Mayor, L. Casadomé, D. Azuara et al., "Long-range epigenetic silencing at 2q14.2 affects most human colorectal cancers and may have application as a non-invasive biomarker of disease," British Journal of Cancer, vol. 100, no. 10, pp. 1534-1539, 2009.

[27] J. Devaney, C. Stirzaker, W. Qu et al., "Epigenetic deregulation across chromosome 2q14.2 differentiates normal from prostate cancer and provides a regional panel of novel DNA methylation cancer biomarkers," Cancer Epidemiology Biomarkers \& Prevention, vol. 20, no. 1, pp. 148-159, 2011.

[28] X. Wu, T. A. Rauch, X. Zhong et al., "CpG island hypermethylation in human astrocytomas," Cancer Research, vol. 70, no. 7, pp. 2718-2727, 2010.
[29] H. J. Kim, R. A. Barnitz, T. Kreslavsky et al., "Stable inhibitory activity of regulatory $\mathrm{T}$ cells requires the transcription factor Helios," Science, vol. 350, no. 6258, pp. 334-339, 2015.

[30] A. M. Thornton, P. E. Korty, D. Q. Tran et al., "Expression of helios, an ikaros transcription factor family member, differentiates thymic-derived from peripherally induced Foxp3+ T regulatory cells," The Journal of Immunology, vol. 184, no. 7, pp. 3433-3441, 2010.

[31] S. M. Park, H. Cho, A. M. Thornton et al., "IKZF2 drives leukemia stem cell self-renewal and inhibits myeloid differentiation," Cell Stem Cell, vol. 24, no. 1, pp. 153-165, 2019.

[32] Y. Zhao, D. Yun, X. Zou et al., "Whole exome-wide association study identifies a missense variant in SLC2A4RG associated with glioblastoma risk," American Journal of Cancer Research, vol. 7, pp. 1937-1947, 2017.

[33] Y. Wang, S. Yang, Q. Ni et al., "Overexpression of forkhead box J2 can decrease the migration of breast cancer cells," Journal of Cellular Biochemistry, vol. 113, no. 8, pp. 27292737, 2012.

[34] E. Chiarella, A. Aloisio, S. Scicchitano et al., "ZNF521 represses osteoblastic differentiation in human adipose-derived stem cells," International Journal of Molecular Sciences, vol. 19, no. 12, p. 4095, 2018. 